\section{RMD Open}

Rheumatic \&

Musculoskeletal Diseases

\title{
Biological agents for rheumatic diseases in the outbreak of COVID-19: friend or foe?
}

\author{
Cristiana Sieiro Santos (10 , 'Xenia Cásas Férnandez, ${ }^{2}$ Clara Moriano Morales, ${ }^{1}$ \\ Elvira Díez Álvarez, ${ }^{1}$ Carolina Álvarez Castro, ${ }^{1}$ Alejandra López Robles, ${ }^{1}$ \\ Trinidad Pérez Sandoval ${ }^{1}$
}

To cite: Santos CS, Férnandez XC, Moriano Morales C, et al. Biological agents for rheumatic diseases in the outbreak of COVID-19: friend or foe?. RMD Open 2021;7:e001439. doi:10.1136/ rmdopen-2020-001439

Received 4 September 2020 Revised 2 November 2020 Accepted 28 December 2020

Check for updates

(C) Author(s) (or their employer(s)) 2021. Re-use permitted under CC BY-NC. No commercial re-use. See rights and permissions. Published by BMJ.

${ }^{1}$ Rheumatology, Complejo Asistencial Universitario de León, Leon, Castilla y León, Spain ${ }^{2}$ Pharmacy, Complejo Asistencial Universitario de León, Leon, Spain

Correspondence to Dr Cristiana Sieiro Santos; cristysieirosantos@gmail.com

\section{ABSTRACT}

Background The recent outbreak of COVID-19 has raised concerns in the rheumatology community about the management of immunosuppressed patients diagnosed with inflammatory rheumatic diseases. It is not clear whether the use of biological agents may suppose a risk or protection against SARS-CoV-2 infection; however, it has been suggested that severe respiratory forms of COVID-19 occur as a result of exacerbated inflammation status and cytokine production. This prompted the use of interleukin 6 (IL-6) (tocilizumab and sarilumab) and IL-1 inhibitors (anakinra) in severe COVID-19 disease and more recently JAK1/2 inhibitor (baricitinib). Therefore, patients with rheumatic diseases provide a great opportunity to learn about the use of biological agents as protective drugs against SARS-CoV-2.

Objectives To estimate COVID-19 infection rate in patients treated with biological disease-modifying antirheumatic drugs (bDMARDs) for inflammatory rheumatic diseases (RMD), determine the influence of biological agents treatment as risk or protective factors and study the prognosis of patients with rheumatic diseases receiving biological agents compared to the general population in a third-level hospital setting in León, Spain.

Methods We performed a retrospective observational study including patients seen at our rheumatology department who received bDMARDs for rheumatic diseases between December 1st 2019 and December 1st 2020, and analysed COVID-19 infection rate. All patients who attended our rheumatology outpatient clinic with diagnosis of inflammatory rheumatic disease receiving treatment with biological agents were included. Main variable was the hospital admission related to COVID-19. The covariates were age, sex, comorbidities, biological agent, duration of treatment, mean dose of glucocorticoids and need for intensive care unit. We performed an univariate and multivariate logistic regression models to assess risk factors of COVID-19 infection.

Results There were a total of 4464 patients with COVID-19 requiring hospitalisation. 40 patients out of a total of 820 patients with rheumatic diseases $(4.8 \%)$ receiving bDMARDs contracted COVID-19 and 4 required hospital care. Crude incidence rate of COVID-19 requiring hospital care among the general population was $3.6 \%$, and it was $0.89 \%$ among the group with underlying rheumatic diseases. $90 \%$ of patients receiving bDMARDS with
COVID-19 did not require hospitalisation. Out of the 4464 patients, 869 patients died, 2 of which received treatment with biological agents. Patients with rheumatic diseases who tested positive for COVID-19 were older (female: median age 60.8 IQR 46-74; male: median age 61.9 IQR 52-70.3) than those who were negative for COVID-19 (female: median age 58.3 IQR 48-69; male: median age 56.2 IQR 47-66), more likely to have hypertension (45\% vs $26 \%$, 0R 2.25 (Cl 1.18-4.27),p 0.02), cardiovascular disease (23 \% vs 9.6\%, OR $2.73(1.25-5.95)$, p 0.02), be smokers ( $13 \%$ vs $4.6 \%$, OR 2.95 (Cl 1.09-7.98), p 0.04), receiving treatment with rituximab $(20 \%$ vs $8 \%, 2.28$ (Cl 1.24-6.32), $\mathrm{p} 0.02$ ) and a higher dose of glucocorticoids (OR 2.5 (1.3-10.33, p 0.02) and were less likely to be receiving treatment with IL-6 inhibitors $(2.5 \%$ vs $14 \%$, OR 0.16, (Cl 0.10-0.97, p 0.03). When exploring the effect of the rest of the therapies between groups (affected patients vs unaffected), we found no significant differences in bDMARD proportions. IL-1 inhibitors, IL-6 inhibitors, JAK inhibitors and belimumab-treated patients showed the lowest incidence of COVID-19 among adult patients with rheumatic diseases. We found no differences in sex or rheumatological disease between patients who tested positive for COVID-19 and patients who tested negative. Conclusions Overall, the use of biological diseasemodifying antirheumatic drugs (bDMARDs) does not associate with severe manifestations of COVID-19. Patients with rheumatic disease diagnosed with COVID-19 were more likely to be receiving a higher dose of glucocorticoids and treatment with rituximab. IL-6 inhibitors may have a protective effect.

\section{INTRODUCTION}

COVID-19 has spread rapidly across the planet. It is thought to have originated in China's Wuhan province; however, it has spread to more than 140 countries on 6 continents, according to the WHO. ${ }^{1}$

The EULAR and American College of Rheumatology have published preliminary guidance that suggests that patients should discuss treatment discontinuation with their rheumatologists and recommend to temporarily stop 


\section{Key messages}

What is already known about this subject?

- There is no evidence that patients with inflammatory rheumatic diseases (RMD) have a higher risk of SARS-CoV-2 infection nor have a more severe disease course than the general population.

- The Global Rheumatology Alliance identified factores associated with a higher odds of COVID-19 hospitalisation, including older ager, comorbidities and a higher dose of prednisone ( $\geq 10 \mathrm{mg} /$ day) and also found that b/tsDMARD monotherapy was associated with a lower odds of hospitalisation.

What does this study add?

- Patients with inflammatory rheumatic diseases (RMD) treated with biological disease-modifying antirheumatic drugs (bDMARDs) do not seem to present a higher risk of SARS-CoV-2 infection nor a more severe disease course than the general population.

- Patients with RMD with comorbidities such as advanced age, smoking, hypertension and cardiovascular disease have a higher risk of severe SARS-CoV-2 infection, the same risk factors previously identified for COVID-19 infection in general population.

- Most patients with RMD taking immunomodulating therapies recover from COVID-19 infection and do not need to be hospitalised.

- Patients with RMD diagnosed with COVID-19 were more likely to be receiving treatment with rituximab, and receiving a higher dose of glucocorticoids.

- Interleukin 6 inhibitors (IL-6) may protect against COVID-19.

How might this impact on clinical practice or future developments?

- Patients with inflammatory rheumatic diseases diagnosed with COVID-19 were more likely to be receiving treatment with rituximab and glucocorticoids, which should be taken into account by rheumatologists when prescribing treatment for RMD.

- The potential benefict from biological disease-modifying antirheumatic drugs (bDMARDs), particularly IL-6 inhibitors in COVID-19 treatment justifies further studies.

treatment with traditional/biological disease-modifying antirheumatic drugs (bDMARDS) if there is exposure to SARS-CoV-2.

Immunosuppression and comorbidities are associated with an increased risk of serious infection in people with rheumatic diseases; therefore, we might think that people with rheumatic disease may be at higher risk for a more severe course with COVID-19, including hospitalisation, complications and death.

Severe pulmonary and systemic inflammatory manifestations observed in COVID-19 led to a hypothesis about a mechanism of hyperinflammation that depends on the host's response rather than on the direct virusinduced cell damage. ${ }^{2}$ There have been several studies that hypothesised about a preventive therapeutic effect of immunomodulating therapies, such as antimalarials, colchicine, corticosteroids, JAK inhibitors (baricitinib), interleukin 1 (IL-1) inhibitors (anakinra and canakinumab) and interleukin-6 inhibitors (IL-6) (tocilizumab, sarilumab), particularly in patients with COVID-19 who developed pathological immune responses such as cytokine storm. ${ }^{3}$ COVID-19 disease seems to show a higher incidence and severity in patients with risk factors, such as advanced age and some associated comorbidities, mainly hypertension, diabetes, heart disease and previous respiratory diseases. ${ }^{4}$ Based on several studies, there is no overwhelming evidence that patients with rheumatic diseases are at an increased risk. ${ }^{5}$

It remains unknown whether biological diseasemodifying antirheumatic drugs (bDMARDs) pose a higher or lower risk of developing a more severe form of COVID-19 and there is an urgent need for further studies to answer this question.

\section{METHODS}

\section{Study design}

All patients with rheumatic diseases from the rheumatology department at Complejo Asistencial Universitario de León receiving biological disease-modifying antirheumatic drugs (bDMARDs) between the 1st of December 2019 and 1st of December 2020 in the community of León were included. Treatments included in this study were as follows: anti-tumour necrosis factor (TNF) drugs (adalimumab, certolizumab, etanercept, golimumab and infliximab); IL-6 inhibitors (sarilumab and tocilizumab); CTLA4-Ig (abatacept); anti-IL-17 medications (ixekizumab and secukinumab); anti-IL-23 agents (ustekinumab); IL-1 inhibitors (anakinra and canakinumab), JAK inhibitors (baricitinib), BLyS inhibitor (belimumab) and anti-CD20 antibody (rituximab).

We checked for positive reverse transcriptase-PCR on nasopharyngeal and sputum swabs or positive antibodies for SARS-CoV-2 to determine COVID-19 infection rate in patients with rheumatic disease and compared the incidence of patients with rheumatic diseases receiving bDMARDs and the general population. Registry data elements included sociodemographic information, including age and sex; general antecedents such as comorbidities (smoking, hypertension, diabetes mellitus, hyperuricemia, dyslipidaemia, cardiovascular and pneumological comorbidities), type of bDMARD, mean duration of treatment with bDMARD (in months) and mean glucocorticoid dose (mg/day). In the event of COVID-19 infection, we registered the need for hospitalisation, intensive care unit (ICU) and mortality.

\section{Study population}

All adult patients with the following clinical diagnoses: rheumatoid arthritis (RA), psoriatic arthritis (PsA), ankylosing spondylitis (AS), vasculitis (VS), systemic lupus erythematosus (SLE), sjögren (SJ), poly/dermatomyositis (PM/DM), systemic sclerosis (SS) and autoinflammatory syndromes (AI) who were receiving any of the following treatments: anti-TNF alpha drugs (etanercept, adalimumab, infliximab, golimumab and certolizumab), IL-6 inhibitors (tocilizumab and sarilumab), IL-12/23 inhibitors (ustekinumab), IL-17 inhibitors (secukinumab and ixekizumab), IL-1 inhibitors (anankira and canakinumab), CTLA4-Ig (abatacept), JAK inhibitors 
(baricitinib), BLyS inhibitor (belimumab) and anti-CD20 antibody (rituximab) at the time of the study were invited to take part. Duration of treatment with bDMARDS (months) and mean dose of glucocorticoids (mg/day) were also retrieved.

\section{Demographic and clinical data}

The following variables were retrieved from the hospital electronic health record: age, sex, smoking status (smoker, ex-smoker and never smoker), comorbidities (hypertension, diabetes, hyperuricemia, dyslipidaemia, previous lung disease, cardiovascular diseases), rheumatic disease diagnosis, current bDMARDs, duration of treatment with bDMARD (in months) and mean dose of glucocorticoids (mg/day). For this analysis, we considered 'confirmed' cases when the SARS-CoV-2 PCR or antibodies determination for SARS-CoV-2 was performed and resulted positive.

\section{Adherence to treatment}

We checked for treatment adherence within a 5-month period before and after the COVID-19 outbreak and have only included patients who were actively taking their treatment. We used telephone interview and medication disposal registry from our pharmacy department for subcutaneous and intravenous medication. We defined non-adherence as $\geq 20 \%$ change in the dose or frequency of the mentioned medications.

\section{Statistical analysis}

Categorical variables were reported as percentages, whereas continuous variables were expressed as median and IQR values. Quantitative variables were compared by use of the non-parametric Mann-Whitney U test. Categorical variables were compared by use of contingency tables, and $\mathrm{p}$ values were calculated with $\chi 2$ or Fisher's exact tests, when appropriate.

$P$ values less than 0.05 were considered significant. The effect size for retrospective studies was then evaluated with ORs with $95 \%$ CIs.

\section{RESULTS}

In the area of León, there were 820 patients ( $58 \%$ women and $42 \%$ men) who received bDMARDs prescribed for rheumatic diseases during the COVID-19 pandemic. Out of this total, 389 patients (47\%) were diagnosed with RA, 208 patients $(25 \%)$ were diagnosed with AS, 129 (15.7\%) with PsA, 44 (5.4\%) with VS, 22 patients (2.7\%) with SLE, $7(0.85 \%)$ with SJ disease, $4(0.49 \%)$ with $\mathrm{PM} / \mathrm{DM}, 10$ patients $(1.2 \%)$ with AI diseases and 7 patients $(0.85 \%)$ with SS. Group therapeutic distribution was as follows: $568(69 \%)$ with anti-TNF drugs, $110(13.4 \%)$ with antiIL-6, $13(1.6 \%)$ with anti-IL-23 agents, 16 (2\%) with anti-IL-17 agents, $20(2.4 \%)$ with abatacept, $3(0.37 \%)$ with bariticinib, $2(0.24 \%)$ with anti-IL-1, $16(2 \%)$ with belimumab and $72(8.8 \%)$ with rituximab. Most patients had important comorbidities: 226 patients $(27.5 \%)$ had hypertension, 227 (36.1\%) had dyslipidaemia, 84 (10\%) had cardiovascular disease and $57(7 \%)$ had pulmonary disease. A total of 4464 COVID-19 cases required hospital care in the community of León and there were $869(19 \%)$ deaths. Out of the 820 patients with rheumatic diseases receiving bDMARDs, 40 patients $(4.8 \%)$ had COVID-19, $4(0.48 \%)$ required hospitalisation and 2 $(0.24 \%)$ died. COVID-19 crude incidence rate requiring hospital care in the general population was 3.6\%, while in the biological therapy cohort it was $0.48 \%$. The demographic and clinical characteristics of the 820 cases in our registry are shown in table 1 . Patients who tested positive for COVID-19 (female: median age 60.8 IQR 46-74; male: median age 61.9 IQR 52-70.3) than those who were negative for COVID-19 (female: median age 58.3 IQR 48-69; male: median age 56.2 IQR 47-66), more likely to have hypertension ( $45 \%$ vs $26 \%$, OR 2.25 (CI $1.18-4.27)$,p 0.02$)$, cardiovascular disease (23\% vs $9.6 \%$, OR 2.73 (1.25-5.95), p 0.02), be smokers ( $13 \%$ vs $4.6 \%$, OR 2.95 (CI 1.09-7.98), p 0.04), receiving treatment with rituximab (20\% vs $8 \%, 2.28$ (CI 1.24-6.32), p 0.02) and a higher dose of glucocorticoids (OR 2.5 (1.3-10.33, p 0.02 ) and were less likely to be receiving treatment with IL-6 inhibitors $(2.5 \%$ vs $14 \%$, OR 0.16 , (CI $0.10-0.97$,p 0.03 ). When exploring the effect of the rest of the therapies between groups (affected patients vs unaffected), we found no significant differences in bDMARD proportions. IL-1 inhibitors, IL-6 inhibitors, JAK inhibitors and belimumab-treated patients showed the lowest incidence of COVID-19 among adult patients with rheumatic diseases. We found no differences in sex and rheumatological disease between patients who tested positive for COVID-19 and patients who tested negative. Out of the total 820 patients receiving bDMARDs, only 4 of them $(0.48 \%), 3$ men and 1 woman, had COVID-19 requiring hospital care. Two of the patients were receiving treatment with anti-TNF agents, one for RA and the other for PsA, and the other two were receiving abatacept and rituximab for RA. Two patients with abatacept and rituximab died from COVID-19, both with several comorbidities, and both required ICU care. The patient receiving abatacept had administered the last dose of the bDMARD 2-3 weeks before admission and the patient receiving rituximab had his last dosage 1 month prior to the admission. Out of the 40 patients who tested positive for COVID$19,90 \%$ of the cases successfully recovered with no need for hospitalisation. Most patients had a contact with a confirmed or suspected case. To control for possible confounding variables, sequential multivariate regression analyses were performed. In the multivariate analysis, the following risk factors were associated with testing positive for COVID-19: age $>65$ years, smokingcardiovascular disease, which coincided with the univariate analysis.

We also checked for adherence to treatment within 5 months before and after COVID-19 outbreak. The data from this study showed that medication non-adherence was not common within 5 months' period before and after the issue of COVID-19, with only a $10 \%$ of nonadherence during the COVID-19 outbreak. 
Table 1 Demographic and clinical characteristics of 820 RMD treated with bDMARDs in our registry

\begin{tabular}{|c|c|c|c|c|c|}
\hline & Total & $\begin{array}{l}\text { COVID-19 } \\
\text { Positive }\end{array}$ & $\begin{array}{l}\text { COVID-19 } \\
\text { Negative }\end{array}$ & & \\
\hline & $n=820$ & $n=40$ & $\mathrm{~N}=780$ & OR (95\% Cl) & $P$ value \\
\hline Hospitalised & - & 4 & - & - & - \\
\hline ICU care & 2 & 2 & - & - & - \\
\hline Dead & 2 & 2 & - & - & - \\
\hline \multicolumn{6}{|l|}{ Age (years) } \\
\hline Male & $56.15(47-66)$ & $61.9(52-70.3)$ & $56.2(47-66)$ & $1.45(1.35-5.07)$ & 0.03 \\
\hline Female & $58.3(48-69)$ & $60.8(46-74)$ & $58.3(48-69)$ & & \\
\hline \multicolumn{6}{|l|}{ Disease type } \\
\hline RA & 389 & 17 (42.5\%) & $372(47.7 \%)$ & $0.82(0.43-1.56)$ & 0.63 \\
\hline SLE & 22 & 0 & $22(2.8 \%)$ & $0.86(0.11-6.55)$ & 0.62 \\
\hline AS & 208 & $9(22.5 \%)$ & 199 (25.5\%) & $0.85(0.41-1.81)$ & 0.85 \\
\hline PsA & 129 & $9(22.5 \%)$ & $120(15.4 \%)$ & $1.6(0.74-3.44)$ & 0.26 \\
\hline SJ & 7 & $2(5 \%)$ & $5(0.64 \%)$ & $8.16(1.53-43.42)$ & 0.06 \\
\hline VS & 42 & $3(7.5 \%)$ & $39(5 \%)$ & $1.54(0.45-5.22)$ & 0.45 \\
\hline SS & 7 & 0 & $7(0.89 \%)$ & 1.71 (0.09 to 30.68$)$ & 1 \\
\hline PM/DM & 4 & 0 & $4(0.51 \%)$ & 1.72 (0.09 to 30.79$)$ & 0.71 \\
\hline $\mathrm{Al}$ & 10 & 0 & $10(1.3 \%)$ & 1.22 (0.07 to 21.28$)$ & 0.89 \\
\hline \multicolumn{6}{|l|}{ Comorbidities } \\
\hline $\mathrm{HT}$ & 226 & $18(45 \%)$ & $208(26 \%)$ & $2.25(1.18-4.27)$ & 0.02 \\
\hline $\mathrm{HU}$ & 49 & $5(12.5 \%)$ & $44(5.6 \%)$ & $2.39(9.89-6.4)$ & 0.08 \\
\hline DL & 227 & 15 (37.5\%) & $212(27.2 \%)$ & $1.61(0.83-3.11)$ & 0.20 \\
\hline DM & 73 & $6(15 \%)$ & $67(8.6 \%)$ & $1.88(0.76-4.63)$ & 0.16 \\
\hline$C D$ & 84 & $9(23 \%)$ & 75 (9.6\%) & $2.73(1.25-5.95)$ & 0.02 \\
\hline PD & 57 & $5(12.5 \%)$ & $52(6.7 \%)$ & $2(0.75-5.32)$ & 0.19 \\
\hline \multicolumn{6}{|l|}{ Smoking } \\
\hline Active & 41 & $5(13 \%)$ & $36(4.6 \%)$ & $2.95(1.09-7.98)$ & 0.04 \\
\hline Ex-smokers & 36 & $5(12.5 \%)$ & $31(3.9 \%)$ & $3.45(1.27-9.42)$ & 0.03 \\
\hline Never & 743 & $20(50 \%)$ & 723 (92.7\%) & $0.08(0.04-0.15)$ & 0.001 \\
\hline \multicolumn{6}{|l|}{ Treatment } \\
\hline Anti-TNF-alpha & 568 & $28(70 \%)$ & $540(69.2 \%)$ & $1.04(0.52-2.07)$ & 1 \\
\hline Anti-IL-1 & 2 & 0 & $2(0.26 \%)$ & 8.62 (0.34 to 216.18$)$ & 0.19 \\
\hline Anti-IL-6 & 110 & $1(2.5 \%)$ & $109(14 \%)$ & $0.16(0.10-0.97)$ & 0.03 \\
\hline Anti-IL-12/23 & 13 & $2(5 \%)$ & $11(1.4 \%)$ & $3.68(0.79-17.19)$ & 0.13 \\
\hline Anti-IL-17 & 16 & $1(2.5 \%)$ & $15(1.9 \%)$ & $1.22(0.16-9.47)$ & 0.56 \\
\hline JAK inhibitors & 3 & 0 & $3(0.38 \%)$ & 1.72 (0.09 to 30.83$)$ & 0.71 \\
\hline Abatacept & 20 & $2(5 \%)$ & $18(2.3 \%)$ & 2.23 (0.5-9.95) & 0.25 \\
\hline Rituximab & 72 & $8(20 \%)$ & $64(8 \%)$ & $2.28(1.24-6.32)$ & 0.02 \\
\hline Belimumab & 16 & 0 & $16(2.1 \%)$ & 0.77 (0.04 to 13.13$)$ & 0.85 \\
\hline \multicolumn{6}{|l|}{ Glucocorticoids } \\
\hline Mean dosage (mg/day) & $10.5(6-13.2)$ & $12.8(6.2-15.8)$ & $9.5(5.5-13.2)$ & $2.5(1.3-10.33)$ & 0.02 \\
\hline $\begin{array}{l}\text { Duration of bDMARDs treatment } \\
\text { (months) }\end{array}$ & $12(7-16)$ & $6.3(2.9-8.3)$ & $15.7(7.2-19.5)$ & $0.44(0.29-0.78)$ & 0.04 \\
\hline
\end{tabular}




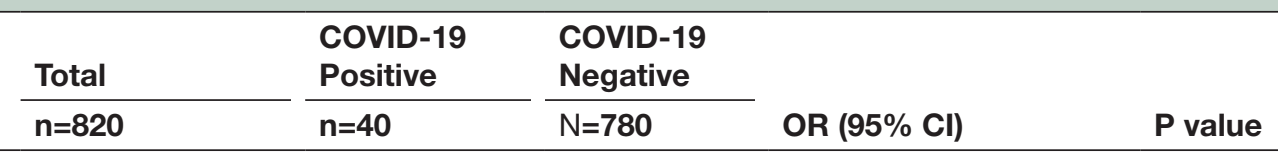

Data are median (IQR) or $\mathrm{n}(\%)$.

Al, autoinflammatory disease; anti-TNF, anti-tumour necrosis factor; AS, ankylosing spondylitis; bDMARDs, biological disease-modifying antirheumatic drugs; CD, cardiovascular ; DL, dyslipidaemia; DM, diabetes mellitus; HT, hypertension; HU, hyperuricemia; ICU, intensive care unit; IL, interleukin; PD, pulmonary disease; PM/DM, polymyositis/dermatomyositis; PsA, psoriatic arthritis; RA, rheumatoid arthritis; SJ, Sjogren's disease; SLE, systemic lupus erythematosus; SS, systemic sclerosis; VS, vasculitis.

\section{DISCUSSION}

Over the last months, Spain has become one of the European countries with more confirmed cases of COVID-19 infection. Rheumatologists have expressed their concerns about patients receiving targeted immune-modulating therapies and have wondered whether patients with underlying immune conditions are most susceptible to contract COVID-19 and have a higher mortality and complications rate.

This work describes epidemiological search of the COVID-19 incidence in patients with rheumatic diseases receiving bDMARDs therapy from a third-level hospital in León, Spain. From a total of 820 patients with immunemediated inflammatory disease treated with bDMARDs, we identified 40 confirmed COVID-19 cases in adults.

We identified factors associated with COVID-19 infection in patients with underlying rheumatic diseases, including age, sex, comorbidities, rheumatic diagnosis, treatment for rheumatic disease, duration of treatment, hospital admission, need for ICU care and mortality rate.

The potential benefit of biological medications in treating COVID-19 is evidenced by those with more severe disease having higher levels of cytokines, including IL-6 and TNF. The use of IL-6 inhibitors is being investigated for COVID-19, particularly in cases complicated by aberrant inflammatory responses or 'cytokine storm'. Several studies have theorised that SARS-CoV-2 infection can induce cytokine release, leading to an increase in IL-6, IL-10 and TNF. In cytokine-release syndrome, common laboratory abnormalities in hospitalised patients involve elevated liver enzymes, ferritin value, $\mathrm{C}$ reactive protein, D-dimers, coagulation times (prothrombin time/partial thromboplastin time) and lactate dehydrogenase. ${ }^{4}$

$\mathrm{Xu}$ et al have recently analysed changes of clinical manifestations, CT lung scan and laboratorial results of patients with COVID-19 treated with tocilizumab symptoms and showed that hypoxaemia and CT opacity changes improved immediately after the treatment. ${ }^{5} \mathrm{~A}$ recent study published in The Lancet Rheumatology showed that anakinra reduced both need for invasive mechanical ventilation in the ICU and mortality among patients with severe forms of COVID-19, without serious side effects. ${ }^{6} \mathrm{JAK}$ inhibitors, such as baricitinib, have also been indicated as a possible treatment for COVID-19 by having high affinity of AAK1, a regulator of endocytosis associated with the passage of virus of SARS-CoV-2 into the cell. ${ }^{7}$
Recently, the Global Rheumatology Alliance has published the largest collection of COVID-19 cases among patients with rheumatic diseases, with 600 cases from 40 countries. They identified factors associated with higher odds of COVID-19 hospitalisation, including older age, presence of comorbidities and higher doses of prednisone ( $\geq 10 \mathrm{mg} /$ day), and found that bDMARD/ targeted synthetic DMARD monotherapy was associated with a lower odds of hospitalisation, an effect that was largely driven by anti-TNF therapies. ${ }^{8}$

A retrospective study from Monti and Montecucco showed that none of the 700 patients hospitalised due to severe COVID-19 were receiving biological agents or synthetic therapy, suggesting that patients with immunomodulating therapy are not at a greater risk when compared to the general population. ${ }^{9}$

Our study shows that there is a lower incidence of COVID-19 in the cohort of patients receiving bDMARDs than in general population. Furthermore, this finding is reinforced by the fact that the mean age of patients who developed COVID-19 in the cohort with biological therapy was older than the mean age of patients negative for COVID-19, and several studies have stated that age is an important risk factor for developing a severe form of the disease. ${ }^{1011}$

When comparing the two groups, patients testing positive for COVID-19 were more likely to have a previous diagnosis of hypertension and cardiovascular disease than patients testing negative, although there was no difference in other comorbidities between the two groups, which could seem a contradiction however, it is possible that the comorbidities could influence the severity of the disease, rather than the probability of infection.

In our study, patients testing positive for COVID-19 were more likely to be receiving treatment with rituximab. We searched the literature and found several case studies about patients receiving rituximab that had tested positive for COVID-19 and had suffered a more severe form of the disease. ${ }^{12}$ It has been suggested that the B-cell depletion induced by rituximab could reduce the immunogenicity of several vaccines and have a negative effect in immunocompromised patients who are more susceptible to develop COVID-19. ${ }^{1311}$

We also found that patients with COVID-19 were less likely to be receiving treatment with IL- 6 inhibitors, reinforcing the idea that IL-6 inhibitors such as tocilizumab and sarilumab could protect against SARS-CoV-2, 
which seems reasonable as they are being used as treatment for pneumonia in patients with severe COVID-19. While analysing the duration of treatment, we found that patients who tested negative for COVID-19 have been receiving treatment with bDMARDs for a longer period of time than patients who were positive, which could be interpreted as argument in favour of the protective role of biological agents in COVID-19. While analysing the dose of glucocorticoids, we found a significant difference between the two groups of patients, which may indicate that a higher dose of corticosteroids are a risk factor for serious infection, including COVID-19.

It would be of great interest to clarify if patients with immunosuppressive therapy are more likely to contract COVID-19 than the general population; and in case of contracting COVID-19, if the biological therapies suppose a higher rate of complications such as secondary bacterial pneumonia or acute respiratory distress syndrome. However, it seems that the host's innate immune system is the main driver of pulmonary inflammation. ${ }^{1415}$

We also did not find significant differences in sex and rheumatological disease between patients who tested positive for COVID-19 and patients who tested negative.

We should acknowledge some limitations in our study that might limit definite conclusions: (1) a low number of COVID-19 cases in patients with rheumatic disease; (2) asymptomatic patients with no PCR test or serology test were considered negative; (3) the patients receiving biological agents are relatively young (mean age for man 56.15 and 58.3 for women); (4) a low number of patients receiving treatment with IL-12/23, IL-1, JAK inhibitors and belimumab; (5) patients with rheumatic diseases treated with immunosuppressive treatment or biological agents are more likely to pay attention to hygienic and distance measures than the general population.

Overall, the prognosis of patients with rheumatic diseases with COVID-19 is good; however, our findings should be interpreted with caution. Our study's results suggest that patients receiving biological therapy for underlying rheumatic diseases do not show a higher risk for developing severe manifestations of COVID-19 and that the risk is very likely to be lower than the general population. Also, according to our previous study, comorbidities such as hypertension, dyslipidaemia, diabetes and interstitial lung disease, and age seem to be two of the most determinant risk factors of developing a severe form of the disease. ${ }^{1516}$

\section{CONCLUSION}

Overall, the use of bDMARDs does not associate with severe manifestations of COVID-19. Patients with rheumatic disease diagnosed with COVID-19 were more likely to be receiving treatment with rituximab and a higher dose of glucocorticoids. IL-6 inhibitors may have a protective effect against them when compared with the general population.
Acknowledgements The authors acknowledge the assistance of study participants, radiographers, study nurses and laboratory staff who participated in the study.

Contributors CSS and XCF conceived the presented idea. CSS and CMM performed the computations. CM verified the analytical methods. CSS, CMM, EAD, $\mathrm{CAC}$ and ALR supervised the findings of this work. All authors discussed the results and contributed to the final manuscript.

Funding The authors have not declared a specific grant for this research from any funding agency in the public, commercial or not-for-profit sectors.

Competing interests None declared.

Patient consent for publication Not required.

Ethics approval The study was conducted according to the principles of the Declaration of Helsinki and approved by the Institutional Ethics Committee of Complejo Asistencial Universitario de León.

Provenance and peer review Not commissioned; externally peer reviewed.

Data availability statement All data relevant to the study are included in the article or uploaded as supplemental information. All data relevant to the study are included in the article.

Open access This is an open access article distributed in accordance with the Creative Commons Attribution Non Commercial (CC BY-NC 4.0) license, which permits others to distribute, remix, adapt, build upon this work non-commercially, and license their derivative works on different terms, provided the original work is properly cited, appropriate credit is given, any changes made indicated, and the use is non-commercial. See: http://creativecommons.org/licenses/by-nc/4.0/.

ORCID iD

Cristiana Sieiro Santos http://orcid.org/0000-0003-0889-9877

\section{REFERENCES}

1 Pan F, Ye T, Sun P, et al. Time course of lung changes at chest CT during recovery from coronavirus disease 2019 (COVID-19). Radiology 2020;295:715-21.

2 Henderson LA, Canna SW, Schulert GS, et al. On the alert for cytokine storm: immunopathology in COVID-19. Arthritis Rheumatol 2020;72:1059-63.

3 Mehta P, McAuley DF, Brown M, et al. COVID-19: consider cytokine storm syndromes and immunosuppression. Lancet 2020;395:1033-4.

4 Chen N, Zhou M, Dong X, et al. Epidemiological and clinical characteristics of 99 cases of 2019 novel coronavirus pneumonia in Wuhan, China: a descriptive study. Lancet 2020;395:507-13.

$5 \mathrm{Xu}$ X, Han M, Li T, et al. Effective treatment of severe COVID-19 patients with tocilizumab. Proc Natl Acad Sci U S A 2020;117:10970-5.

6 Huet T, Beaussier H, Voisin O, et al. Anakinra for severe forms of COVID-19: a cohort study. Lancet Rheumatol 2020;2:e393-400.

7 Richardson P, Griffin I, Tucker C, et al. Baricitinib as potential treatment for 2019-nCoV acute respiratory disease. Lancet 2020;395:e30-1.

8 Gianfrancesco M, Hyrich KL, Al-Adely S, et al. Characteristics associated with hospitalisation for COVID-19 in people with rheumatic disease: data from the COVID-19 global rheumatology alliance physician-reported registry. Ann Rheum Dis 2020;79:859-66.

9 Monti S, Montecucco C. Prevalence of COVID-19 among patients with rheumatic diseases: the need to await results from large collaborative studies. Response to: 'COVID-19 pneumonia in a large cohort of patients treated with biological and targeted synthetic antirheumatic drugs' by Conticini et al. Ann Rheum Dis 2020:annrheumdis-2020-217738.

10 Wang D, Hu B, Hu C, et al. Clinical characteristics of 138 hospitalized patients with 2019 novel coronavirus-infected pneumonia in Wuhan, China. JAMA 2020;323:1061.

11 Messina F, Pampaloni F, Piaserico S. Comment on: recovery from COVID-19 in a patient with spondyloarthritis treated with TNFalpha inhibitor etanercept. A report on a patient with COVID-19 with psoriatic arthritis receiving ustekinumab. Ann Rheum Dis 2020:annrheumdis-2020-218029.

12 Schulze-Koops H, Krueger K, Vallbracht I, et al. Increased risk for severe COVID-19 in patients with inflammatory rheumatic diseases treated with rituximab. Ann Rheum Dis 2020:annrheumdis-2020-218075.

13 Guilpain P, Le Bihan C, Foulongne V, et al. Rituximab for granulomatosis with polyangiitis in the pandemic of covid-19: 
lessons from a case with severe pneumonia. Ann Rheum Dis 2021;80:e10.

14 Prete M, Favoino E, Catacchio G, et al. SARS-CoV-2 inflammatory syndrome. clinical features and rationale for immunological treatment. Int J Mol Sci 2020;21. doi:10.3390/ijms21093377. [Epub ahead of print: 10 May 2020].
15 Figueroa-Parra G, Aguirre-Garcia GM, Gamboa-Alonso CM, et al. Are my patients with rheumatic diseases at higher risk of COVID-19? Ann Rheum Dis 2020;79:pii: annrheumdis-2020-217322.

16 Santos CS, Morales CM, Álvarez ED, et al. Determinants of COVID-19 disease severity in patients with underlying rheumatic disease. Clin Rheumatol 2020;39:2789-96. 